KUNS-1408

$\mathrm{HE}(\mathrm{TH}) 96 / 09$

August 1994

\title{
Gribov Copy and Complex Phase of Chiral Determinant*
}

\author{
T. Aoyama ${ }^{a}$ and Y. Kikukawa ${ }^{a}$ \\ ${ }^{\text {a }}$ Department of Physics, Kyoto University, Kyoto 606-01, Japan
}

We calculate the complex phase of chiral determinant by the vacuum overlap formula with configurations of two-dimensional U(1) gauge field fixed in Landau and Laplacian gauge. The complex phase fluctuates over the Gribov copies, which appear in the process of Landau gauge fixing and contain vortex-like singularities. In the Laplacian gauge, the fluctuation can be reduced and the phase can be determined uniquely. If it is used as a preconditioning for Landau gauge fixing, the most smooth configuration is obtained among the copies generated.

\section{Introduction}

For a smooth background gauge field, the vacuum overlap formula 1 [ 6 gives a definite and well-defined method to calculate the complex phase of chiral fermion determinant in lattice regularization. The complex phase is gauge noninvariant by this method. Its variation under a gauge transformation was shown to give the consistent anomaly 1, [7 9]. In anomaly-free U(1) chiral gauge theories on the two-dimensional torus, the complex phase by the vacuum overlap formula reproduces the exact result in the continuum limit with uniform gauge fields [2].

For generic gauge fields on the lattice, however, it was argued by several authors 10, 11, 4.,6 that the nature of gauge degrees of freedom could spoil the chiral structure of the formula. For the $(\mathrm{D}+1)$ dimensional system of Wilson fermion with kink mass and the wave guide of gauge interaction 12], it was shown 10] that the gauge fluctuation induces another light fermion mode of opposite chirality at the boundary of the wave guide. Such a light mode could give the contribution to the complex phase of chiral determinant.

On the other hand, it was shown[6] that the singular gauge transformation which wipe out the large uniform vector potential into the singular one with delta function, breaks the gauge invariance of chiral determinant. This happens in the anomaly-free $\mathrm{U}(1)$ chiral gauge theories on the two-dimensional torus in the continuum limit, provided that the anti-periodic boundary

\footnotetext{
*Talk presented by Y. Kikukawa at Lattice'96, St. Louis,
} USA, 4-8 June 1996. condition is chosen for all fermions. The vacuum overlap reproduces the result as well. The authors of ref. [6] claimed that it is these singular gauge transformations which spoils the chiral phase when averaged on the gauge orbit.

In this article, we report our results of numerical study about the gauge dependence of chiral determinant in two-dimensional $\mathrm{U}(1)$ chiral gauge theory. First we present our result on the gauge integration of chiral determinant. We choose a small uniform vector potential as background. For this value, the chiral determinant in the continuum limit is gauge invariant even under the singular gauge transformation mentioned above[6]. Next we calculate the complex phase of chiral determinant with the gauge field configurations fixed in Landau gauge. We examine the effect of the singularity associated to the Gribov copy 13, 14. Finally, we examine the performance of Laplacian gauge fixing, especially as a preconditioning for Landau gauge 15. .

We consider the anomaly free $\mathrm{U}(1)$ chiral gauge theory with four right-handed fermions with unit charge and one left-handed fermion with charge two, which we call "11112" model following ref. [6]. The boundary condition is anti-periodic for all fermions. The lattice size is $L=8$ and 16. The small uniform vector potential (toron) is chosen as $A_{1} L / 2 \pi=0.20$ and $A_{2} L / 2 \pi=0.15$. Generic U(1) gauge fields are generated at the value of the gauge coupling $e L / \pi=0.05$ by the Metropolis algorithm. 


\section{Gauge Integration}

With the toron in consideration, the complex phase of the determinant for the "11112" model takes the following value on the lattice $L=16$,

$\phi_{0}=0.13304$.

Gauge functions, $g(n)_{k}(k=1, \ldots, N)$ are randomly generated and the variations of the complex phases by the gauge transformations, $\phi_{i}-\phi_{0}$ are calculated. Then the complex phase $\phi$ of the gauge-integrated determinant is calculated as

$$
\exp (\Delta S+i \phi)=\left\langle\exp \left(i \phi_{k}\right)\right\rangle \text {. }
$$

In Figure 1, we show a result for $N=15,000$. We plot the averaged value for each bin of 750 gauge transformations and also the accumulated average over 750 times the number of bins $I$.

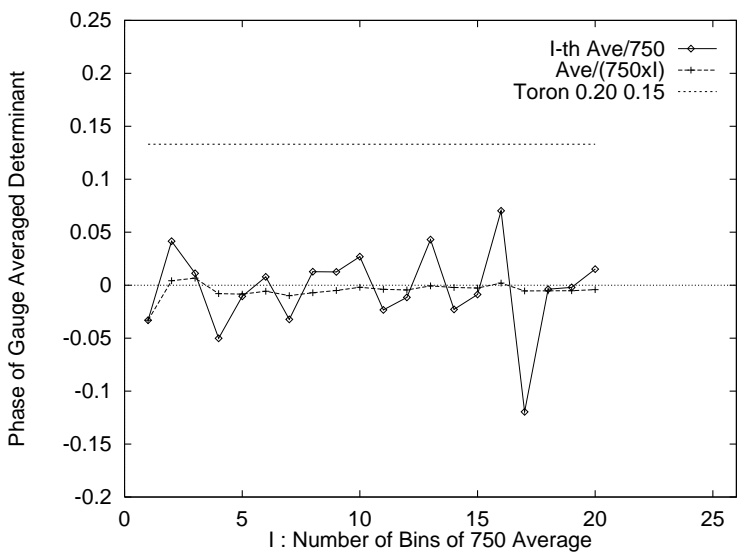

Figure 1. Complex phase of chiral determinant averaged over $N=15,000$ gauge functions.

We repeated such measurement three times and obtained

$\phi=-0.0035 \pm 0.006$

The standard error is used in this estimate. The result shows that the large gauge fluctuation affects the chiral phase even for the small toron.

\section{Gauge Fixing and Chiral Determinant}

We first show, in Figure 2, a typical gauge potential of the Gribov copy of the toron in consideration, which is generated by the usual local algorithm for Landau gauge fixing. The gauge function which generates such a copy from the original toron is also shown. We can clearly observe the vortex singularity 14 .
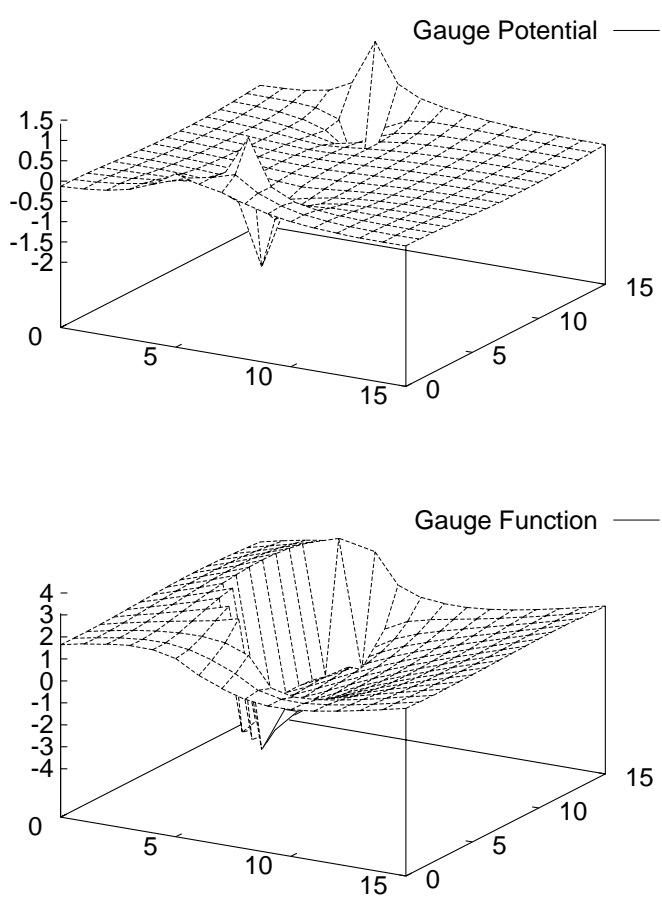

Figure 2. Vector potential and gauge function of a Gribov copy of the toron, $A_{1} L / 2 \pi=0.2$, $A_{1} L / 2 \pi=0.15 . L=16$.

In Figure 3, we show the complex phases calculated in Landau gauge for a generic $\mathrm{U}(1)$ gauge field at $e L / \pi=0.05$. We can observe the fluctuation of the complex phases. It is clear that the singularity in the gauge function associated to the Gribov copy affect the complex phase very much 13.

The complex phases calculated in Laplacian gauge are also shown in Figure 3. This gauge reduces the fluctuation of gauge degree of freedom completely and gives a unique complex phase on the gauge orbit. The result of its use as the preconditioning is also impressive. It gives the most smooth configuration among the Gribov copies generated, as far as we examined. The obtained value of the complex phase is also reasonable. 

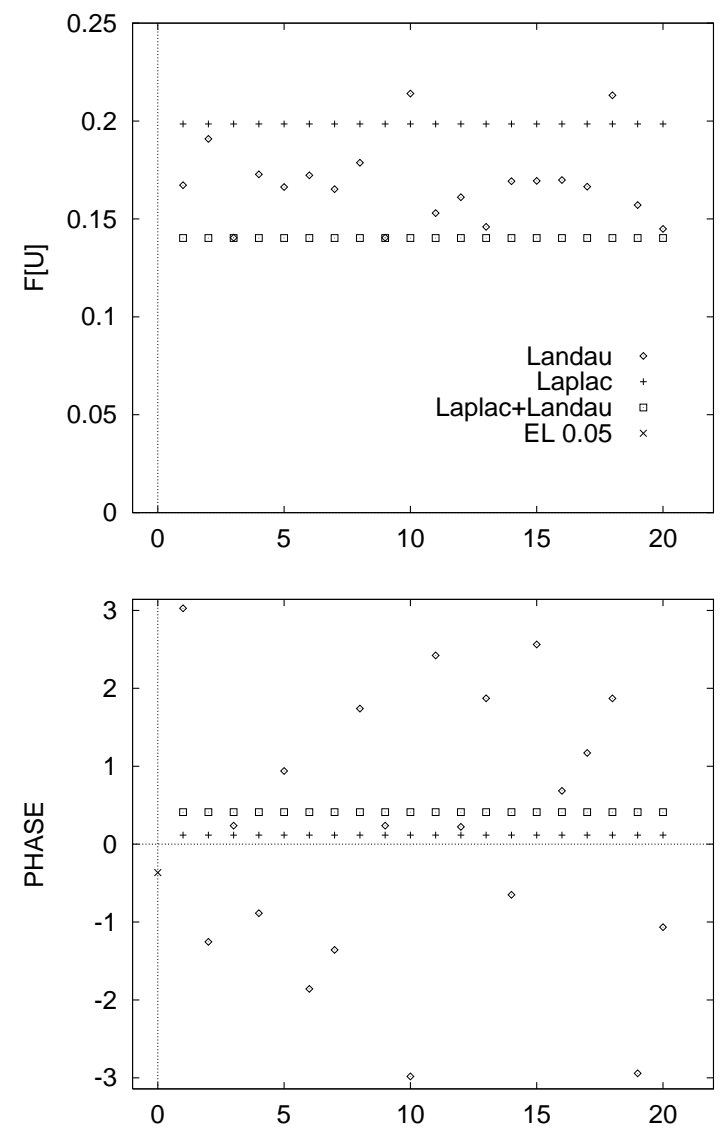

Figure 3. Minimized values of $F[U]$ and complex phases of chiral determinant for Gribov copies generated from a generic electric field at $e L / \pi=$ 0.05. 20 successive random gauge transformations are applied. $L=8$.

\section{Conclusion}

The complex phase defined by the vacuum overlap formula is very sensitive to the singular gauge functions associated to the Gribov copies. It is hard to determine a unique phase on a gauge orbit by gauge-fixing into Landau gauge with usual local algorithm. The Laplacian gauge fixing reduces the gauge fluctuation completely and gives a unique phase. If the gauge coupling constant is small enough, the fixed configuration is rather smooth. Preconditioning for Landau gauge fixing by the Laplacian method is practically useful because, as far as we examined, it gives the most smooth configurations among the Gribov copies.

This work is supported by National Laboratory for High Energy Physics, as KEK Supercomputer Project (Project No. 10).

\section{REFERENCES}

1. R. Narayanan and H. Neuberger, Nucl. Phys. B412 (1994) 574; Phys. Rev. Lett. 71 (1993) 3251; Nucl. Phys. B34(Proc. Suppl.) (1994) 95, 587; Nucl. Phys. B443 (1995) 305.

2. R. Narayanan and H. Neuberger, Phys. Lett. B348 (1995) 549.

3. R. Narayanan, H. Neuberger and P. Vranas, Phys. Lett. B353 (1995) 507.

4. R. Narayanan and H. Neuberger, Phys. Lett. B358 (1995) 303.

5. P. Huet, R. Narayanan and H. Neuberger, Phys. Lett. B380 (1995) 291.

6. R. Narayanan and H. Neuberger, UW-PT96-04, Mar 1996.

7. Y. Shamir, Nucl. Phys. B417 (1993) 167.

8. S. Aoki and R.B. Levien, Phys. Rev. D51 (1995) 3790.

9. S. Randjbar-Daemi and J. Strathdee, Phys. Lett. B348 (1995) 543; Nucl. Phys. B443 (1995) 386.

10. M.F.L. Golterman, K. Jansen, D.N. Petcher and J. Vink, Phys. Rev. D49 (1994) 1606; Nucl. Phys. B34(Proc. Suppl.), 1994; M.F.L. Golterman and Y. Shamir, Phys. Rev. D51 (1995) 3026.

11. M.F.L. Golterman and Y. Shamir, Phys. Lett. B353 (1995) 84; Erratum-ibid. B359 (1995) 422.

12. D.B. Kaplan, Phys. Lett. B288 (1992) 342; Nucl. Phys. B30(Proc. Suppl.) (1993) 597.

13. Y. Shamir, Nucl. Phys. B47(Proc. Suppl.) (1996) 212.

14. Ph. de Focrand and J. Hetrick, Nucl. Phys. B42(Proc. Suppl.) (1995) 861.

15. J.C. Vink and U-J. Wiese, Phys. Lett. B289 (1992) 122; J.C. Vink, Phys. Lett. B321 (1994) 239; Phys. Rev. D51 (1995) 1292. 